$\xi=$

\title{
Estimation of Fugitive Dust Levels on A Construction Site Using Digital Image Information
}

\author{
Im, Sun-Young ${ }^{1}$, Yu, Jung-Ho ${ }^{2} *$ \\ ${ }^{1}$ Master Student, Department of Architecture Engineering, Kwangwoon University, South Korea, isy9754@kw.ac.kr \\ ${ }^{2}$ Professor, Department of Architecture Engineering, Kwangwoon University, South Korea \\ *Corresponding author E-mail: myazure@ kw.ac.kr
}

\begin{abstract}
Currently, fugitive dust management on construction sites does not focus on prevention but rather seeks ways to control it after occurrence. In order to effectively manage fugitive dust on a construction site, it is necessary to quantify prevent fugitive dust from occurring, and take immediate measures after occurrence. Therefore, this study proposes a technique to estimate the amount of fugitive dust generated on a construction site using digital image information. Based on the results of this study, it is expected that high levels of fugitive dust on construction sites can be prevented in the future and efficient management will be possible.
\end{abstract}

Keywords: Fugitive Dust, Construction Site, Light Scattering Method, Digital Image Information Analysis Method

\section{Introduction}

The World Health Organization (WTO) recommends an annual fugitive dust standard of $20 \mu \mathrm{g} / \mathrm{m}^{3}$ and an ultrafine dust standard of $10 \mu \mathrm{g} / \mathrm{m}^{3}$. However, Korea's fugitive dust (PM10) levels average well over this at $46.75 \mu \mathrm{g} / \mathrm{m}^{3}$. This is higher than in cities in other OECD countries such as Los Angeles and Tokyo. In addition, between 2014 and 2016, the ultrafine dust concentration in Seoul was $26 \mu \mathrm{g} / \mathrm{m}^{3}$, which is over twice as high as that of New York and Tokyo. Fugitive dust accounts for $44.3 \%$ of Korea's fugitive dust (PM10), with 22\% coming from construction sites (National Institute of Environmental Research). This indicates that dust emissions from construction sites can have a serious effect on domestic fugitive dust levels and can cause air pollution. Dust emissions from construction sites are regulated by Article 43 of the Air Quality Preservation Act, Article 44 of the Enforcement Decree of the same Act, Article 57, and Article 58 (Actions and installations for the reduction of dust emissions on construction sites). These laws outline specific actions and installations designed to reduce dust levels. However, small construction sites are excluded from these regulations and are checked temporary tasks such as whether there are things such as the installation of dust covers and water cleaning facilities. In other words, there is no specific system for monitoring the level of dust emissions. In the U.S. (specifically, Los Angeles), inspectors monitor for visible dust in the control of fugitive dust. In the U.K. (especially London), there is an automonitoring system for monitoring and controlling fugitive dust based on visibility. It would also be beneficial for Korea to implement a similar system to recognize and quantitatively monitor dust emissions and use this information to reduce dust levels. Thus, this study suggests a method for the automatic monitoring of dust levels on construction sites using digital image information.

\section{Literature Review}

In order to estimate the amount of fugitive dust in the current construction site, previous research using digital image information analysis is insufficient. In order to measure the visibility of the atmosphere among the existing researches, the research using digital image information analysis is similar to the fugitive dust estimation. There are two previous studies that measure the visibility of the atmosphere using digital image information analysis. First, it is a luminance ratio method that compares the brightness difference between digital image information and measures visibility. For the visibility measurement, digital image information analysis was performed using the digital image information measured by installing a reference to a specific object or a narrow area. In Kedu's study, they measured visibility using luminance ratio of plume and around it by taking a picture to plume from chimney (KeDu et 1, 2007). Otherwise, Wangki Yuen focused on measurement method with luminance ratio by comparing the difference between plume and white background (Wangki et 8, 2017). Furthermore, other methods dealt with distance-ratio which can measure distance from target to target in digital image information. This method used digital image information by pointing big object in picture as a reference. Baumer's research developed and suggested an algorithm that city's visibility measurement by using this method. If the set-target would be identified in approximately $60 \mathrm{~km}$, a $70 \mathrm{~km}$ level, is the most visible rank, would be used for visibility in The German Meteorological Centre (Baumer et 1, 2008).

The digital image information analysis technique used in the previous studies was used for narrow or wide area. In the study of the narrow area, the study was carried out with reference to the object with clear distinction of the dust generation part such as the chimney or the white background. In case of a wide area such as cities, 
the research conducted by taking pictures to between the several references which has a clear distance. However, since the boundary between the affected part and the affected part of the dust generated at the construction site is not clear, it is difficult to refer to a reliable object such as a visibility study for a narrow area. In addition, construction sites have limited sites, so it is difficult to refer to distant objects such as visibility studies for large areas. Therefore, it is necessary to develop a new method which is complementary to the method used in previous researches in order to grasp the amount of fugitive dust generated in the limited site of the construction site.

\section{Technique for Estimation Fugitive Dust in Construction Site Using Digital Image In- formation}

A number of variables can affect the measurement of dust emissions from a construction site when using digital image information. For example, the external environment, such as the sun, wind, and weather, can affect the digital images, while different results can be gained depending on where the images are taken from. Therefore, it is desirable to first derive a formula for the calculation of dust emissions through experimentation in a controlled environment that is not affected by external factors. The experimental method for this laboratory set-up is presented in Fig. 1.

The experimental study utilizes a DSLR camera, a dust generator, artificial sunlight, and a dust meter. In the experiment, the correlation between the dust and the image is analyzed at a fixed illuminance value. The illumination and internal environmental conditions are then altered to reflect a real-life environment. The dust generated from the dust generator is photographed from various directions, and the dust is measured using the dust meter. A database is then constructed based on the collected image and dust data. Based on this database, a formula for dust accumulation is derived.

\section{Conclusion}

Most studies related to the measurement of fugitive dust using digital images have been conducted to determine atmospheric fugitive dust on construction sites is based on amelioration after the fact rather than prevention. Therefore, based on the results of this study, it is expected that fugitive dust on construction sites can be reduced and efficient management is possible. In future research, it is necessary to build a database and develop a dust calculation method using the experimental method presented in this study.

\section{Acknowledgement}

This research was supported by a grant(17CTAP-C114926-02) from Infrastructure and transportation technology promotion Program funded by the Ministry of Land, Infrastructure and Transport of Korean government.

This research was supported by a grant (18AUDP-B127891-02) from the Architecture \& Urban Development Research Program funded by the Ministry of Land, Infrastructure and Transport of the Korean government.

\section{References}

[1] Chin-Hsiang Luo, Che-Yen Wen, Chung-Shin Yuan, Jiun-Jian Liaw, Cho-Ching Lo, Shin-Hsuan Chiu, (2005), Investigation of urban atmospheric visibility by high-frequency extraction : Model development and field test, Atmospheric Environment 39, pp25452552

[2] D. Baumer, S. Versick, B. Vogel, (2008), Determination of the visibility using a digital panorama camera, Atmospheric Environment 42, pp2593-2602

[3] KeDu, Mark J.Rood, (2007), Quantification of plume opacity by digital photography, Environ. Sci. Technol., 41 (3), pp 928-935

[4] Nathan Graves, Shawn Newsam, (2011), Using visibility cameras to estimate atmospheric light extinction, Applications of Computer Vision (WACV), pp577-584

[5] Nathan Graves, Shawn Newsam, (2012), visibility cameras ; where and how to look, MAED '12 Proceedings of the 1st ACM international workshop on Multimedia analysis for ecological data pp7-12

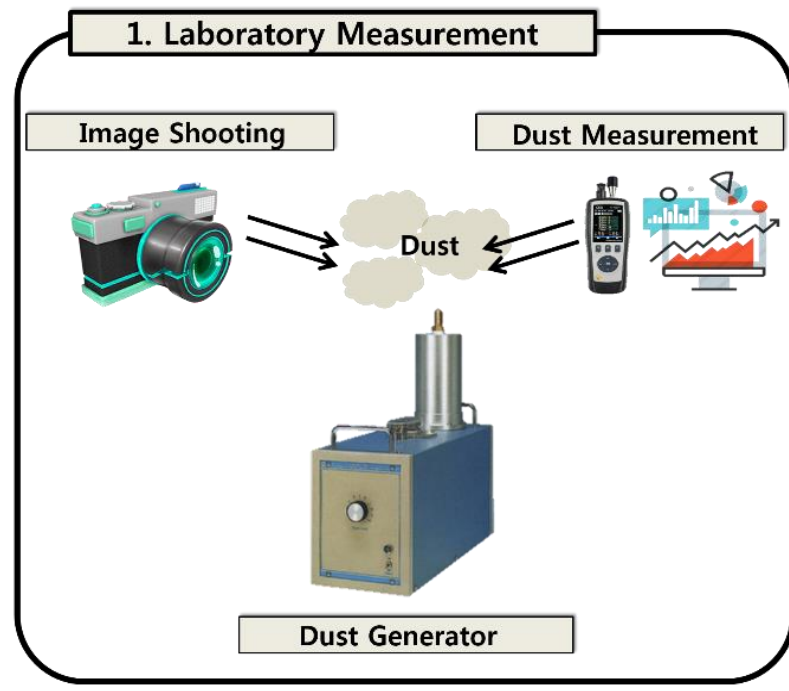

2. Dust Calculation Method Estimation

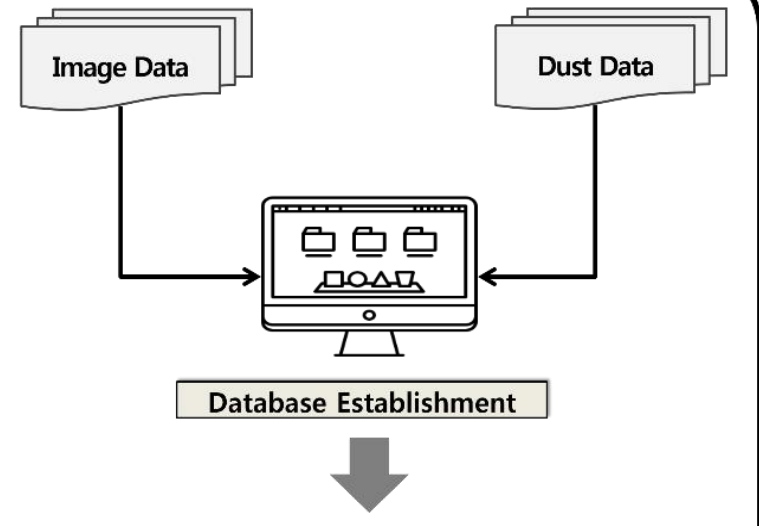

Dust Calculation Method Estimation

Fig . 1: Technique for the estimation of fugitive dust levels in on a construction site using digital image informations visibility for meteorological and atmospheric purposes. Limitations arise when attempting to apply these methods directly to construction sites. Therefore, this study proposed a method to estimate the levels of fugitive dust generated on a construction site using digital image information. Currently, the management of 